\title{
Evaluation of the effects of bovine demineralized bone matrix and coralline hydroxyapatite on radial fracture healing in rabbit
}

\author{
Ali Aliabadi ${ }^{1}$, Arash Esfandiari ${ }^{2}$, Maryam Farahmand ${ }^{2}$, Amirashkan Mahjoor ${ }^{3}$ and \\ Samira Mojaver ${ }^{3}$
}

${ }^{1}$ Department of Clinical Studies, Faculty of Veterinary Medicine, Kazeroon Branch, Islamic Azad University, Kazeroon, Iran.

${ }^{2}$ Department of Basic Science, Faculty of Veterinary Medicine, Kazeroo Branch, Islamic Azad University, Kazeroon, Iran.

${ }^{3}$ Department of Pathobiology, Faculty of Veterinary Medicine, Kazeroon Branch, Islamic Azad University, Kazeroon, Iran.

\section{Accepted 16 March, 2012}

\begin{abstract}
Stimulating bone production may be applied to the management of fractures, non-unions, and osteomyelitis. Hydroxyapatite is compatible and osteoconductive for bone regeneration. This study was conducted to evaluate the effect of osteoinductive activity of xenogenic demineralized bone matrix (DBM) in combination with osteoconductive hydroxyapatite (HA) in osseous location. Twenty four adult New Zealand white rabbits (male, weight: 2.5 to $3.0 \mathrm{~kg}$ ) were randomly divided into four groups. All rabbits were anesthetized with intramuscular administration of ketamin and xylazine. After preparing the operation site, the right radius was exposed, and a full thickness $5 \mathrm{~mm}$ defect was created in the mid-diaphyseal region. The defects were filled with DBM and hydroxyapatite in Group I, DBM in Group II, hydroxyapatite in Group III and Group IV was considered as a control group. The formation and healing of bone were determined by histological and mechanical analysis during 8 weeks. Implantation of combination of DBM and hydroxyapatite yielded significant $(p<0.05)$ bone formation resulting in histological, and mechanical evidence of union compared to other groups. The second group which received DBM showed better bone healing compared to the third group. No instances of union were observed in the hydroxyapatite and control groups. Our results suggest that bovine DBM may be more useful in combination with hydroxyapatite as a therapeutic adjuvant in clinical situations when local formation of bone is needed.
\end{abstract}

Key words: Demineralized bone matrix, coralline hydroxyapatite, bone healing, rabbit.

\section{INTRODUCTION}

Over the last decades, a great deal of research has focused on therapies for enhancing bone repair (Alper et al., 1989). Stimulating bone production may be applied to the management of fractures, non-unions, and osteomyelitis (Cook et al., 1994). Autogenous bone graft is the gold standard among the graft materials because it provides all of these properties. Autogenous bone graft has been the implant of choice for most of the orthopaedic

\footnotetext{
*Corresponding author. E-mail: aaliabadi@gmail.com.
}

procedures. However, autogenous and allogenic bone grafts have several limitations, such as donor-site infection, pain, and disease transfer (Finkemeier, 2002). Because of these limitations, biosynthetic bone graft substitutes are being investigated. Bone graft substitutes should possess one or more of the characteristics typical of autograft: osteoconductivity, osteoinductivity and osteogenicity. Demineralized bone matrix (DBM) has been used for several decades in human surgery for the treatment of nonunion, facial deformities, osteomyelitis, and large defects resulting from benign tumor removal (Ragni et al., 1993). DBM has been shown to aid in the 
stimulation of an osteoinductive response allowing for improved bone growth and fusion. Many studies have also demonstrated the ability of DBM to be completely resorbed and replaced by new bone formation in rat spinal fusion studies (Bomback et al., 2004; Lee et al., 2005; Wang et al., 2007). Hydroxyapatite is compatible and osteoconductive for bone regeneration. Coralline hydroxyapatite is commercially available as ProOsteon 200 and 500 (Interpore Cross International, Irvine, CA). ProOsteon_500R appears to mimic the internal structure of human bone. This synthetic material is made by subjecting a common, nondecorative form of coral (Goniopora sp.) to a patented chemical process, which converts the coral to hydroxyapatite that shares a similar mineral content as that of human bone. A combination of DBM and HA mixture would probably create a composite with both osteoconductive and osteoinductive properties.

The porous, interconnected structure of the coral remains intact, providing an ideal matrix through which new bone tissue can grow. It has demonstrated the ability to aid in the stimulation of an osteoinductive response due to the presence of naturally occurring osteoinductive growth factors. This study was conducted to evaluate the effect of osteoinductive activity of xenogenic DBM in combination with osteoconductive hydroxyapatite in osseous location.

\section{MATERIALS AND METHODS}

The laboratory care, anesthesia, and euthanasia of animals used in this study were performed in accordance with the Guide for the Care and Use of Laboratory Animals. Twenty four skeletally mature New Zealand white rabbits were used in this study (male, weight: 2.5 to $3.0 \mathrm{~kg}$ ), all obtained from the same source, in order to decrease genetic variability. All rabbits were anesthetized with intramuscular administration of ketamin $(40 \mathrm{mg} / \mathrm{kg}$; Alfasan International, Woerden, Holland) and xylazine (13 mg/kg; Alfasan International, Woerden, Holland). The right forelimb was shaved and prepared with Betadine. After preparing the operation site, the right radius was exposed, and an approximately $5 \mathrm{~mm}$ full thickness bone defect was created in the middle diaphyseal region in the radius of right forearm. Rabbits were divided into four groups, Group I was the control group, and the rabbits in this group received no treatment. There are three experimental groups. The segmental defects in Group II were implanted with DBM alone, and Group III were implanted with hydroxyapatite (HA). Group IV were implanted with DBM and HA mixture combination with equal parts. Following implantation, the skin incisions were closed with 3-0 absorbable suture and the skin was closed with 2-0 absorbable suture and sealed with Vetbond. The DBM used for this study was prepared from the bovine long bones in a similar manner as that of human BioSet_RT.

The demineralization process was similar to that employed by Urist (1991). Frozen fragmented bone was grounded to particle 1 $\mathrm{mm}$ in size. The bone matrix was extracted in $4 \mathrm{~mol} \mathrm{GUHCL}$ at $4^{\circ} \mathrm{C}$ for $72 \mathrm{~h}$ after the pulverized bone had been demineralized in $6.6 \mathrm{~N}$ $\mathrm{HCl}$ the extracted solution was passed through millipore filter pore size $0.6 \mathrm{~m}$ (milipore coperation ML. USA). The filtered solution was dialized against deionized water and that water insolubale precipitate was re-desolved in $4 \mathrm{M}$ GUHCL. Gelatin peptides were removed by dialysis against $0.52 \mathrm{~m}$ citrate buffer and the precipitate was centrifuged and lyophilized. The final DBM particle size distribution of between 150 and $850 \mathrm{~nm}$ was obtained by sieving the bone before and after demineralization. Osteoinductivity of each lot was determined in the rabbit ectopic pouch model. Coralline hydroxyapatite (ProOsteon_ 500R; Interpore Cross International, Irvine, CA) was prepared using aseptic technique for extrusion from a syringe in $0.5 \mathrm{cc}$ doses. The defect also filled with $10 \mathrm{mg}$ of liquid DBM $\left(2 \mathrm{mg} / \mathrm{mm}^{2}\right)$. The formation and healing of bone were determined by histological, and mechanical analysis during 8 weeks. For histological examination, the harvested tissues were decalcified with $10 \%$ formic acid solution which was changed daily. The samples were embedded in paraffin, and $5 \mu \mathrm{m}$-thick sections cut through the long axis and stained with hematoxylin and eosin (Gruber and Stasky, 1999). During the histological examination, the pathologist was unaware of the group to which each specimen belonged. For biomechanical evaluation, the samples had undergone the three-point bending test (Amsler FM 2750, Roell + Korthaus, Schaffhausen, Switzerland). And we compared structural stiffness and ultimate load in all groups. Data was analysed with SPSS 11.0 software for Windows with the paired sample test and the Wilcoxon non-parametric two-related sample test.

\section{RESULTS}

Implantation of combination of DBM and hydroxyapatite yielded significant $(p<0.05)$ bone formation resulting in histological, and mechanical evidence of union compare to other groups. Histological grading scale for the degree of bone healing were used to evaluate the union qualification based on quality of union, new bone formation, cortex development and remodeling (Table 1). Histopathological evaluation was shown that quality of the fracture union, cortex development and newbone formation were significantly better $(p<0.05)$ when compared to the other groups (Figure 1). Three other groups turned out to show no significant difference but DBM group shows better healing in some histologic slide compare to HA and control group. Defects were filled with trabeclar bone in HA+DBM group but in the HA and control group there were only a few fibrous tissue observed (Figures 2, 3 and 4). Biomechanical evaluation revealed better result in DBM+HA group compare to other groups. Structural stiffness and ultimate load was significantly increased $(p<0.05)$ in $\mathrm{DBM}+\mathrm{HA}$ group compare to three other groups. There was no significant difference between HA group and control group but DBM group showed significant $(p<0.05)$ difference when compare to HA and control group (Table 2).

\section{DISCUSSION}

Autogenous bone graft in segmental fracture healing is gold standard when compare to other methods, due to some well-known disadvantages, such as the need for the secondary surgical site, donor-site morbidity, infection and quantity-related problem, the search continues for a suitable alternative. Another possibly used bone graft material is an allograft bone which had been found to be less successful when compared to autografts (Hopp et al., 1989; Moore et al., 1989). As a result, some 
Table 1. Histological grading scale for the degree of bone healing.

\begin{tabular}{lll}
\hline & No sign of fibrous or other union & 0 \\
Quality of union and new bone formation & Fibrous union & 1 \\
& Fibrocartilaginous union or cartilage union & 2 \\
& Mineralizing cartilage and bone union & 3 \\
& Bone union & 4 \\
& & 0 \\
& No cortex formed & 1 \\
Cortex development and remodeling & Formation of new bone along exterior borders & 2 \\
& Formation of both the outer cortex border and the medullary space & 3 \\
& Cortices formed but incomplete bridging & 4 \\
\hline
\end{tabular}

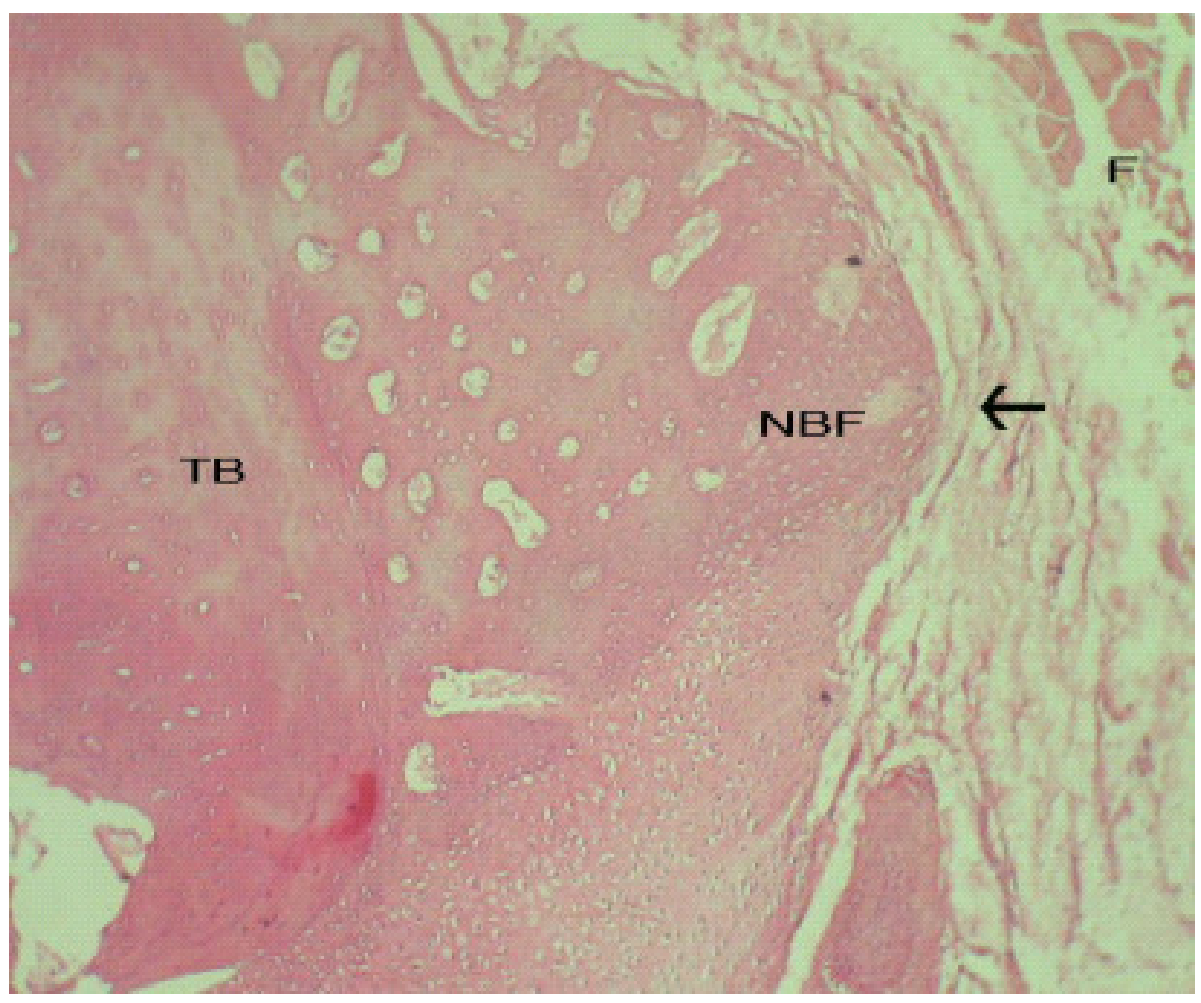

Figure 1. Defect filled with Trabeclar bone and new bone formation in HA+DBM group. (TB: Trabecular bone, NBF: new bone formation, F: fibrous tissue).

investigators, looking for a new bone graft substances such as biologically processed materials, biopolymers, growth factors, and cells to use in similar situations (Parikh, 2002). Ideal bone graft substitutes should possess osteoinductive and osteoconductive properties. DBM is an osteoinductive agent, and has been proved to establish good results for the healing of fractures and bone defects (Bauer and Muschler, 2000; Bingel, 1999; Farso-Nielsen et al., 1992). Delivering this material to the defect site through the use of an scaffolding synthetic material seems logical. Hydroxyapatite derived from chemically converted corals has been used successfully as bone graft substitutes in orthopedic surgeries (Damien et al., 1990; Sartoris et al., 1987). One of the most popular mixtures is the mixture of DBM and HA, which has demonstrated variable results in different studies (Hopp et al., 1989; Moore et al., 1987; Parikh, 2002).

In our study, DBM and the HA mixture were combined to provide better fracture healing. Different results were obtained in similar research projects, whilst some of them supported our results. In our study, the best fracture healing was observed in the mixture of DBM and HA group, and there were no significant differences between the HA and the control group whereas DBM group showed 


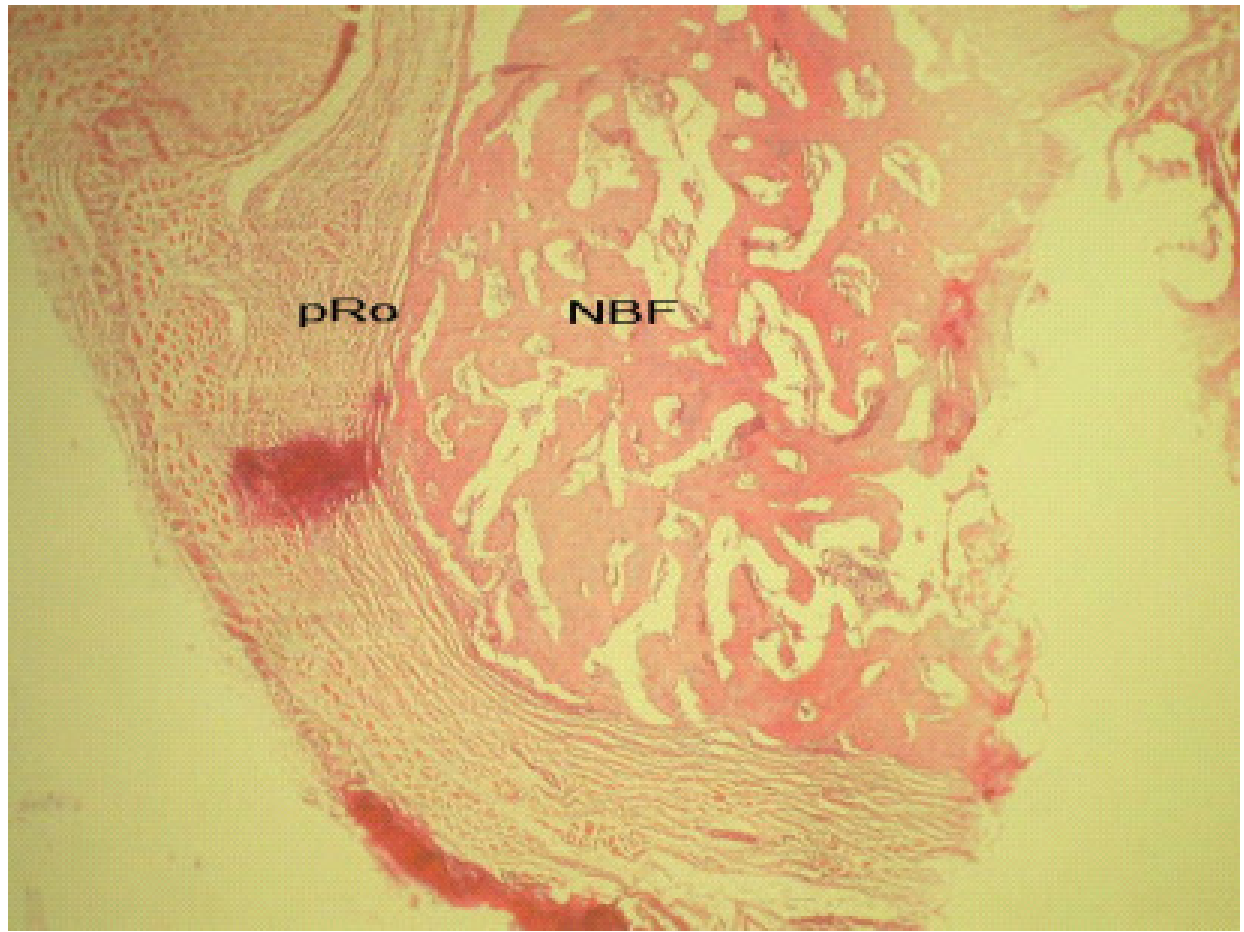

Figure 2. New bone formation in DBM group at the end of experiment (NBF: new bone formation, PRO: preosteum).

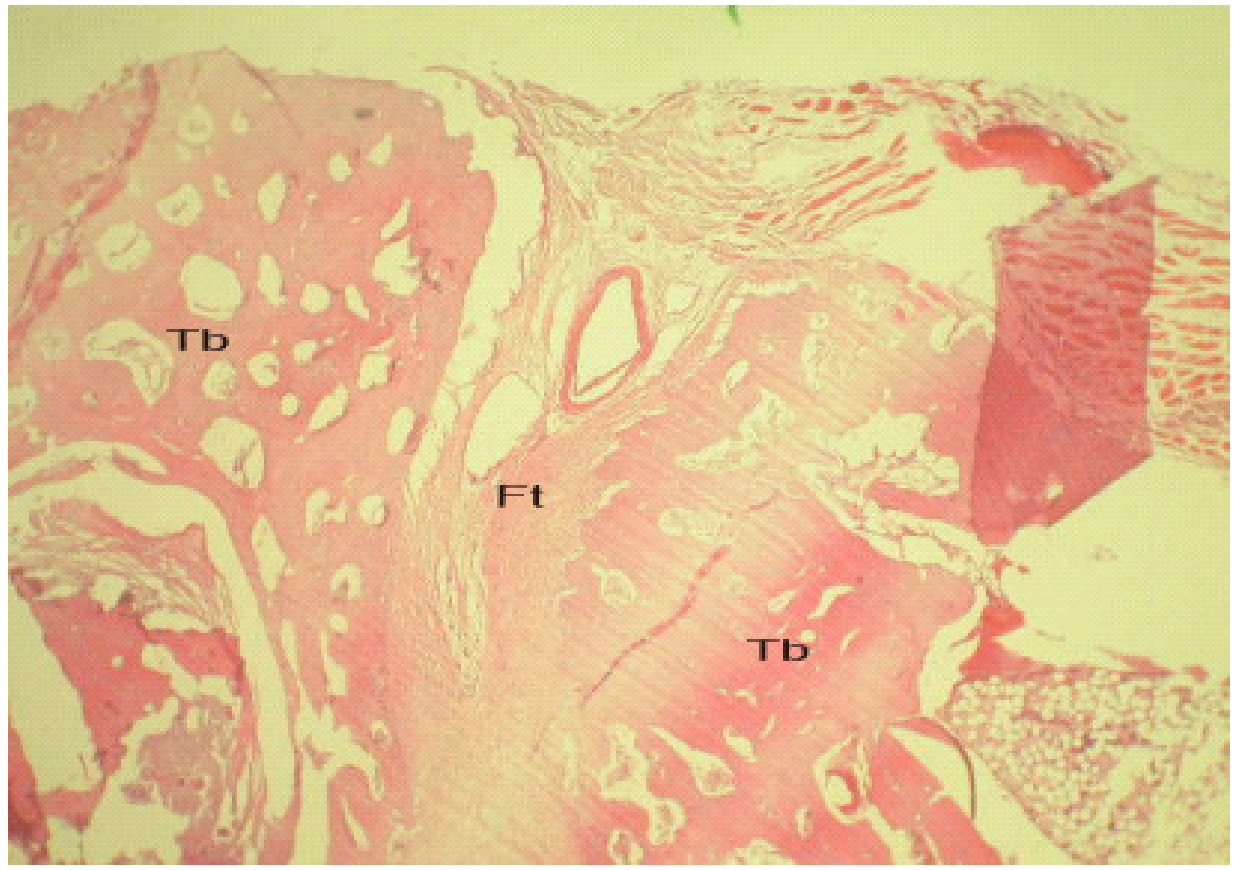

Figure 3. Defect filled with fibrous tissue in HA group at the end of experiment (Ft: Fibrous tissue; Tb: Trabecular bone).

significantly better healing when compare to $\mathrm{HA}$ and control Groups. These data illustrate the osteoconductive properties of HA and osteoinductive capacity of DBM.
Good healing in DBM+HA group may be due to osteoconductive properties of $\mathrm{HA}$ in combination with Osteoinductive DBM. Our findings were found to be 


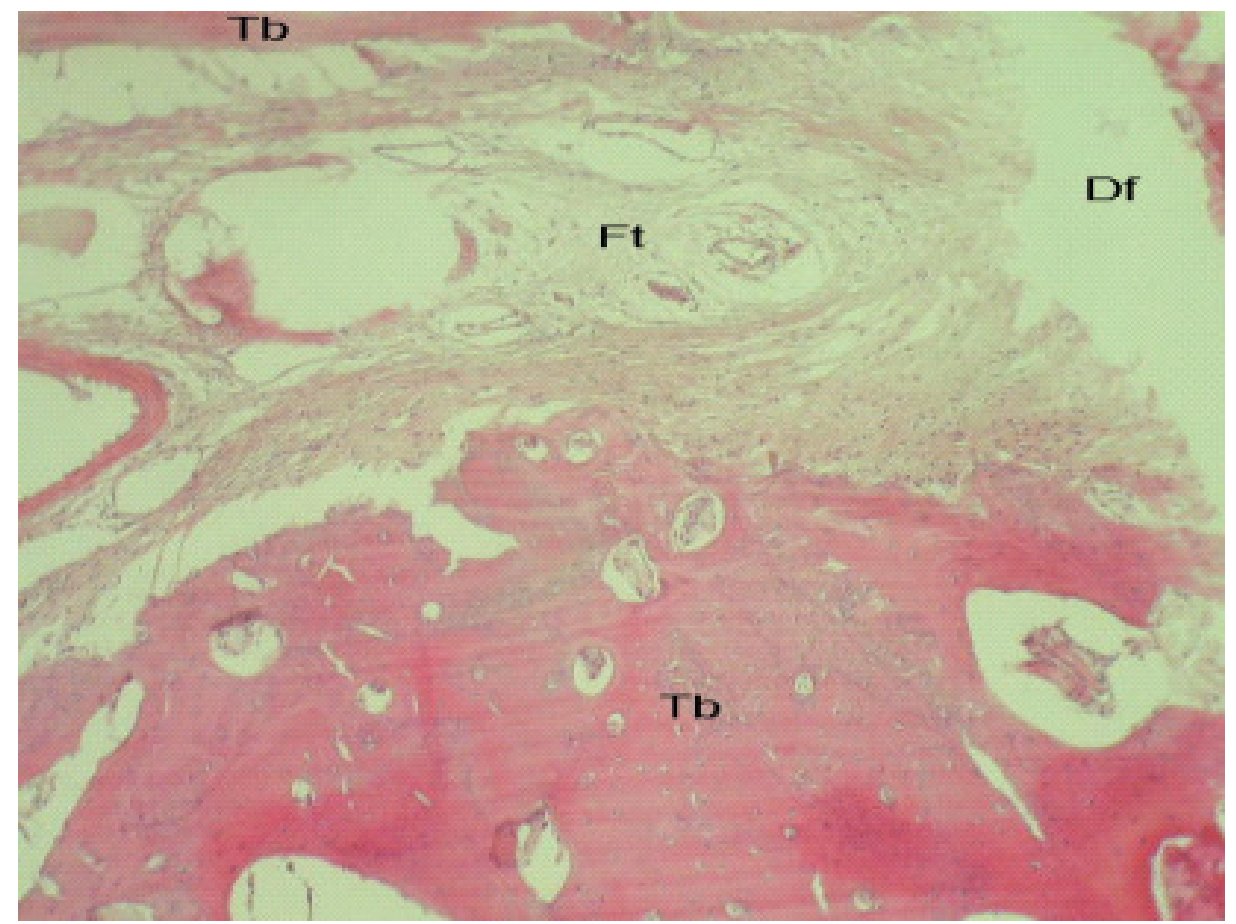

Figure 4. Defect filled with fibrous tissue in control group (Df: Defect, Tb: Trabeclar bone, Ft: fibrous tissue).

Table 2. Biomechanical evaluation results.

\begin{tabular}{lcccc}
\hline Group factor & DBM+HA & DBM & HA & Control \\
\hline Structural stiffness & $8.97 \pm 4.37^{*}$ & $1.08 \pm 0.75$ & $0.80 \pm 0.12$ & $0.50 \pm 0.10$ \\
Ultimate load & $25.20 \pm 5.38^{*}$ & $7.46 \pm 3.00$ & $4.36 \pm 2.79$ & $3.26 \pm 2.80$ \\
\hline
\end{tabular}

similar to some of the earlier studies. Hopp et al. (1989) designed an experiment in which the ulnar defects of rats were grafted by HA, DBM, HA/DBM, autogenous bone graft and allogenic bone graft in five different groups. At the sixth week, they found that the plain HA group scored worse than the control group, whereas all other groups displayed better scores. Ragni and Lindholm (1991) created lumbar intervertebral fusion in rats, they used HA and DBM in combination, and compared the outcomes with three other groups which consisted of HA, DBM and autogenous grafting. After two months, the HA/DBM group provided better fusion than the plain HA and plain DBM groups. In an experiment by Moore et al. (1987) in which they aimed to graft wide ulnar defects of dogs with HA/TCP mixtures. They used mixture of autogenous cancellous bone in equal ratio and HA/TCP mixtures for one of the other groups, and pure autogenous cancellous bone for the control group. At the end of the study they found that six dogs which were only grafted with the HA/TCP mixture showed fibrous nonunion, whereas other groups displayed union.

Finally they suggested that HA/TCP should be used as a mixture with autogenous graft. At the other hand challenging the data provided by the authors above, there are some experiments that are demoting the usage of a combination of HA with other materials. In one study Alper et al. (1989) identified the role of hydroxyapatite (HA) and demineralised bone matrix (DBM) combination in fracture healing. They created $5 \mathrm{~mm}$ segmental defects in the radii of the rats, defects were grafted by DBM, HA and $\mathrm{DBM} / \mathrm{HA}$ mixture. At the end of the second month, radii were investigated histologically, and the HA group was found to have worse results when compared to the control group. They stated that DBM alone was an osteogenetic material for the healing in non-union models of the rats, but that using HA in conjunction caused these effects to fade away. Numerous studies have also demonstrated the ability of DBM to be completely resorbed and replaced by new bone formation in rat spinal fusion studies (Bomback et al., 2004; Lee et al., 2005; Wang et al., 2007), as well as in dogs (Nunamaker, 1998) and rabbits (Leupold et al., 2006). Lee et al. (2005) examined the effect of a combination of DBM and HA on osteogenesis both in vitro and in vivo using an athymic 
nude rat abdominal muscle pouch model, and evaluated the possibility of HA as a carrier of DBM, they proved that DBM/HA putty indicated osteoblastic differentiation in vitro and showed ectopic mineralized tissue formation in the rat abdominal pouch model. These findings indicate that the DBM/HA putty can retain its oteoinductivity and HA can be used as a carrier of DBM (2011). Ragni et al. (1993) used HA, DBM, and autogenous bone marrow for the vertebral fusion model in rabbits. Two months after the operation, it was found that the groups treated with HA blocks displayed results comparable with the autogenous bone grafting group, and showed better healing than the HA+DBM and $\mathrm{HA}+$ bone marrow groups. However, it was found that the porous HA groups did not display similar results. Histological evidence showed that porous HA granules were wrapped with fibrous reaction tissue and weak bone formation. These data illustrate the osteoconductive properties of $\mathrm{HA}$ and also the osteoinductive capacity of DBM has been welldocumented at the end of the second month, the HA/DBM group provided better fusion than the plain HA and plain DBM groups. We concluded that bovine DBM may be more useful in combination with hydroxyapatite as a therapeutic adjuvant in clinical situations when local formation of bone is needed.

\section{REFERENCES}

Alper G, Bernick S, Yazdi M, Nimni ME (1989). Osteogenesis in bone defects in rats: the effects of hydroxyapatite and demineralized bone matrix. Am. J. Med. Sci., 298(6): 371-376.

Bauer TW, Muschler GF (2000). Bone graft materials, An overview of the basic science. Clin. Orthop., 371: 10-27.

Bingel SA (1999). Euthanasia and necropsy. In An YH, Friedman RJ (eds) Animal models in orthopaedic research. CRC Press, Boca Raton, pp. 71-81.

Bomback DA, Grauer JN, Lugo R, Troiano N, Patel T, Friedlaender GE (2004). Comparison of Posterolateral Lumbar Fusion Rates of Grafton Putty and OP-1 Putty in an Athymic Rat Model. Spine, 29: $1612-1617$.

Cook SD, Baffes GC, Wolfe MW, Sampath TK, Rueger DC (1994). Recombinant human bone morphogenetic protein-7 induces healing in a canine long-bone segmental defect model. Clin. Orthop., 301: 302-312.
Damien CJ, Parsons JR, Benedict JJ, Weisman DS (1990). Investigation of a Hydroxyapatite and Calcium Sulfate Composite Supplemented with an Osteoinductive Factor. J. Biomed. Mater. Res., 24: 639-654.

Farso-Nielsen F, Karring T, Gogolewski S (1992). Biodegradable guide for bone regeneration. Polyurethane membranes tested in rabbit radius defects. Acta. Orthop. Scand., 63: 66-69.

Finkemeier CG (2002). Bone-grafting and bone-graft substitutes. J. Bone Joint Surg. Am., 84(3): 454-464.

Gruber HE, Stasky AA (1999). Histologic study in orthopaedic animal research. In: An YH, Friedman RJ (eds) Animal models in orthopaedic research. CRC Press, Boca Raton, pp. 115-137.

Hopp SG, Dahners LE, Gilbert JA (1989). A study of the mechanical strength of long bone defects treated with various bone autograft substitutes: an experimental investigation in the rabbit. J. Orthop. Res., 7(4): 579-584.

Lee YP, Jo M, Luna M, Chien B, Lieberman JR, Wang JC (2005). The Efficacy of Different Commercially Available Demineralized Bone Matrix Substances in an Athymic Rat Model, J. Spinal Disord. Tech., 18: $439-444$

Leupold JA, Barfield WR, An YH, Hartsock LA (2006). A Comparison of ProOsteon, DBX, and Collagraft in a Rabbit Model., J. Biomed. Mater. Res., B Appl. Biomater., 79: 292-297.

Moore DC, Chapman MW, Manske D (1987). The evaluation of a biphasic calcium phosphate ceramic for use in grafting longbone diaphyseal defects. J. Orthop. Res. 5(3): 356-365.

Nunamaker DM (1998). Experimental models of fracture repair. Clin. Orthop., 355: S56-S65.

Parikh SN (2002). Bone graft substitutes in modern orthopedics. Orthopedics, 25(11): 1301-1309.

Ragni P, Ala-Mononen P, Lindholm TS (1993). Spinal fusion induced by porous hydroxyapatite blocks (HA). Experimental comparative study with $\mathrm{HA}$, demineralized bone matrix and autogenous bone marrow. Ital. J. Orthop. Traumatol., 19(1): 133-144.

Ragni P, Lindholm TS (1991). Interaction of allogeneic demineralized bone matrix and porous hydroxyapatite bioceramics in lumbar interbody fusion in rabbits. Clin. Orthop., 272: 292-299.

Sartoris DJ, Holmes RE, Bucholz RW, Mooney V, Resnick D (1987). Coralline Hydroxyapatite Bone-graft Substitutes in a Canine Diaphyseal Defect Model. Radiographic-histometric Correlation, Invest. Radiol., 22: 590-596.

Urist (1991). Interaction of allogeneic demineralized bone matrix and porous hydroxyapatite bioceramics in lumbar interbody fusion in rabbits. Clin. Orthop., 272: 292-299.

Wang JC, Alanay A, Mark D, Kanim LEA, Campbell PA, Dawson EG, Lieberman JR (2007). A Comparison of Commercially Available Demineralized Bone Matrix for Spinal Fusion, Eur. Spine. J., 16: 1233-1240. 